\title{
ON GROUPS OF EXPONENT FOUR. III
}

\author{
N. D. GUPTA AND R. B. QUINTANA, JR.
}

\begin{abstract}
C. R. B. Wright has shown that the nilpotency class of an n-generator group of exponent four is at most $3 n-1$. In this paper, it is shown that if this bound can be improved to $3 n-3$ then the free group of exponent four of infinite rank is solvable.
\end{abstract}

Introduction. Let $\kappa(n)$ and $\lambda(n)$ denote the nilpotency class and the solvability length respectively of the free $n$-generator group of exponent 4 . If $\mu(n)$ denotes the nilpotency class of the free $n$ generator metabelian group of exponent 4 , then $\mu(n)=n+1$ if $n \geqq 4$ and $\mu(n)=n+2$ if $n=2,3$ (Gupta-Tobin [1]). Since $\kappa(n) \geqq \mu(n), \kappa(n) \rightarrow \infty$ as $n \rightarrow \infty$. On the other hand, Marshall Hall, Jr. (verbal communication) and G. Higman [4] have conjectured that there exists an integer $l$ such that $\lambda(n) \leqq l$ for all $n=$ $2,3, \cdots$. In this connection, the reader is also referred to Tritter [5] and Gupta-Weston [2]. It is a well-known result of C. R. B. Wright [6] that $\kappa(n) \leqq 3 n-1$. In this paper, we establish a connection between the HallHigman conjecture and the exact value of $\kappa(n)$. Our main result is as follows:

THEOREM. If $\lambda(k) \rightarrow \infty$ as $k \rightarrow \infty$, then for $n \geqq 3, \kappa(n)=3 n-2$ or $3 n-1$.

Notation. $[x, y]=x^{-1} y^{-1} x y ;[x, y, z]=[[x, y], z] ; \gamma_{m}(G)$ is the $m$ th term of the lower central series of $G ;\left\langle x^{G}\right\rangle$ is the normal closure of $x$ in $G$. A commutator is of type $(r \rightarrow s)$ if it is of weight $s$ in $r$ variables.

Preliminaries. In this section, we shall state and prove a number of lemmas required for the proof of the main result.

LEMMA 1. Let $n$ be a fixed integer exceeding 2 and let $G$ be a group of exponent 4 in which every commutator of type $(n \rightarrow 3 n-2)$ is trivial. For each $m \geqq 0$, let $C_{m}=\left[x_{i(1)}, y_{1}, \cdots, y_{m}, x_{i(2)}, \cdots, x_{i(3 n-3)}\right]$ where $|\{i(1), \cdots, i(3 n-3)\}| \leqq n-1$. Then $C_{m} \in \gamma_{4}\left(\left\langle x_{i(j)}^{G} !\right)\right.$ for some $j=1,2, \cdots$, $3 n-3$.

Proof. Since $|\{i(1), \cdots, i(3 n-3)\}| \leqq n-1$, we may assume that for some $r$ and $s, 1<r<s \leqq 3 n-3, i(1)=i(r)=i(s)$. We now argue that 
$C_{m} \in \gamma_{4}\left(\left\langle x_{i(1)}^{G}\right\rangle\right)$. Since $\left[x_{i(1)}, y_{1}, \cdots, y_{m}\right] \in\left\langle x_{i(1)}^{G}\right\rangle$, we have $\left[x_{i(1)}, y_{1}, \cdots, y_{m}\right]=$ $\prod_{j=1}^{t} x_{i(1)}^{\varepsilon_{j} z_{j}}$ where $\varepsilon_{j} \in\{-1,1\}$, and $z_{j} \in G$. Thus in turn we have

$$
\begin{aligned}
C_{m} & =\left[\prod_{j=1}^{t} x_{i(1)}^{\varepsilon_{j} j}, x_{i(2)}, \cdots, x_{i(3 n-3)}\right] \\
& =\prod_{j=1}^{t}\left[x_{i(1)}^{z_{j}}, x_{i(2)}, \cdots, x_{i(3 n-3)}\right]^{e_{j}} \bmod \gamma_{4}\left(\left\langle x_{i(1)}^{G}\right\rangle\right)
\end{aligned}
$$

(since $i(1)=i(r)=i(s)$ for some $r, s, 1<r<s)$. Further, since $x_{i(1)}^{z_{1}}=$ $x_{i(1)}\left[x_{i(1)}, z_{j}\right]$, we have

$\left[x_{i(1)}^{z_{1}}, x_{i(2)}, \cdots, x_{i(3 n-3)}\right]$

$$
=\left[x_{i(1)}, \cdots, x_{i(3 n-3)}\right]\left[x_{i(1)}, z_{j}, x_{i(2)}, \cdots, x_{i(3 n-3)}\right] \bmod \gamma_{4}\left(\left\langle x_{i(1)}^{G}\right\rangle\right) .
$$

But the first factor on the right-hand side is of type $(n-1 \rightarrow 3 n-3)$ and is trivial by the result of Wright; and the second factor is of type $(n \rightarrow 3 n-2)$ and is trivial by hypothesis.

Lemma 2 (WRIGHT [6]). Let $G$ be a group of exponent 4 and let $n \geqq 2$, $r \geqq 1$ and $p \geqq 0$ be fixed integers. Then

$$
\begin{aligned}
& {\left[u, x, y, x, x, v_{1}, \cdots, v_{r}, w_{1}, \cdots, w_{s}\right]} \\
& \quad=\left[u, y, v_{1}, \cdots, v_{r-1}, x, v_{r}, x, x, w_{1}, \cdots, w_{s}\right] \bmod \gamma_{n}
\end{aligned}
$$

for all $u \in \gamma_{n}(G)$ and all $x, y, v_{1}, \cdots, v_{r}, w_{1}, \cdots, w_{s} \in G$ (this lemma is obtained by repeated application of congruences (8) and (9) of [6]).

LeMMA 3 (WRIGHT [6]). Let $G$ be a group of exponent 4 and let $i(r)=$ $i(s)=i(t)=q$ for some integers $r, s$, and $t$ with $2<r<s<t \leqq n$. Then modulo $\gamma_{n+1}(G),\left[x_{i(1)}, \cdots, x_{i(n)}\right]$ can be expressed as a product of commutators of the form

and

$$
\left[y_{j(1)}, \cdots, y_{j(n-4)}, x_{q}, y_{j(n-3)}, x_{q}, x_{q}\right]
$$

$$
\left[y_{j(1)}, \cdots, y_{j(n-3)}, x_{q}, x_{q}, x_{q}\right]
$$

where the $y_{j}$ 's are the $x_{i}$ 's in some order.

LEMMA 4 (WRIGHT [6]). Let $G$ be a group of exponent 4 and let $n \geqq 1$ and $m \geqq 0$ be fixed integers. Then modulo $\gamma_{n+m+2}(G),\left[x_{1}, \cdots, x_{n}, y, z_{1}, \cdots, z_{m}\right]$ can be expressed as a product of commutators of the form $\left[y, x_{1 \sigma}, \cdots\right.$, $\left.x_{n \sigma}, z_{1}, \cdots, z_{m}\right]$ where $\sigma$ is a permutation of $\{1, \cdots, n\}$.

LEMMA 5. Let $G$ be a group of exponent 4 and let $C$ be a commutator of type $(k+m \rightarrow 3 k+2 m+l)$ where $k \geqq 3, m \geqq 0, l \geqq 0$. Then modulo $\gamma_{3 k+2 m+l+1}(G)$, $C$ can be written as a product of commutators of the form

$$
\left[x_{i(1)}, y_{1}, \cdots, y_{2 m+l+6}, x_{i(2)}, \cdots, x_{i(3 k-6)}\right]
$$


where $|\{i(1), \cdots, i(3 k-6)\}|=k-2$ and $\left\{y_{1}, \cdots, y_{2 m+l+6}\right\}$ consists of the remaining $m+2$ variables.

Proof. ${ }^{1}$ By Theorem 1 of Wright [6], we may assume that no commutator contains an entry more than 3 times. We prove the lemma by induction on $k \geqq 3$. If $k=3$, there are at least 3 entries in $C$ which appear 3 times and one of them, say $x_{i(1)}$, is not one of the first two entries of $C$. Thus by Lemmas 3 and $4, C$ can be writter, as a product of commutators of the form

$$
\left[x_{i(1)}, y_{1}, \cdots, y_{2 m+l+6}, x_{i(1)}, x_{i(1)}\right],
$$

where $\left\{y_{1}, \cdots, y_{2 m+l+\beta}\right\}$ consists of the remaining $m+2$ variables. Now let $k>3$ and assume that the result has been proved for $k-1$. Write $3 k+$ $2 m+l=3(k-1)+2(m+1) \div(l+1)$. By the induction hypothesis modulo $\gamma_{3(k-1)+2(m+1)+(l+1)+1}(G), C$ can be written as a product of commutators of the form

$$
\left[x_{i(1)}, y_{1}, \cdots, y_{2 m \dashv i+9}, x_{i(2)}, \cdots, x_{i(3 k-9)}\right]
$$

where $|\{i(1), \cdots, i(3 k-9)\}|=k-3$ and $\left\{y_{1}, \cdots, y_{2 m+l+9}\right\}$ consists of the remaining $(m+1)+2$ variables. Thus there are at least 3 variables which appear 3 times in $\left\{y_{1}, \cdots, y_{2 m+l+9}\right\}$ and one of them, say $x_{i(p)}$, is different from $y_{1}$. By Lemma 3, modulo $\gamma_{2 m+l+11}(G)$,

$$
\left[x_{i(1)}, y_{1}, \cdots, y_{2 m+l+8}\right]
$$

is a product of commutators of the form

$$
\left[z_{1}, \cdots, z_{2 m+l+6}, x_{i(p)}, z_{2 m+l+7}, x_{i(p)}, x_{i(p)}\right]
$$

and $\left[z_{1}, \cdots, z_{2 m+l+7}, x_{i(p)}, x_{i(p)}, x_{i(p)}\right]$. Thus modulo $\gamma_{3 k+2 m+l+1}(G)$, using Lemma 2 to the appropriate form, $C$ can be written as a product of commutators of the form

$$
\left[z_{1}, \cdots, z_{2 m+l+7}, x_{j(2)}, \cdots, x_{j(3 k-6)}\right],
$$

where $|\{j(2), \cdots, j(3 k-6)\}|=k-2, \quad x_{i(1)}=x_{j(1)} \in\left\{z_{1}, \cdots, z_{2 m+l+7}\right\}$ and $\left\{z_{1}, \cdots, z_{2 m+l+7}\right\} \backslash\left\{x_{j(1)}\right\}$ consists of the remaining $m+2$ variables. A final application of Lemma 4 gives the desired result.

LEMMA 6. Let $n \geqq 2$ be a fixed positive integer and let $G$ be a group of exponent 4 such that

(i) every commutator of type $(n \rightarrow 3 n-2)$ is trivial,

(ii) $\gamma_{4}\left(\left\langle x^{G},\right)=\{1\}\right.$ for all $x$ in $G$.

Then for all $m \geqq 2$, every commutator of type $(m \rightarrow 2 m+n+1)$ is trivial.

\footnotetext{
${ }^{1}$ We thank the referee for pointing out an oversight in an earlier proof of Lemma 5.
} 
Proof. Let $G$ be generated by $m$ elements $x_{1}, \cdots, x_{m}$. Since $G$ is nilpotent, we may assume that $\gamma_{2 m+n+2}(G)=\{1\}$ and conclude that $\gamma_{2 m+n+1}(G)=\{1\}$. If $m \leqq n+1$, then by the result of Wright, $\gamma_{2 m+n+1}(G)=$ $\{1\}$. Thus we may assume that $m \geqq n+2$. Let $C$ be a typical generator of $\gamma_{2 m+n+1}(G)$. Then $C$ is a commutator of weight $2 m+n+1$ with entries from $x_{1}, \cdots, x_{m}$. Since $C$ is of type

$$
((n+1)+(m-n-1) \rightarrow 3(n+1)+2(m-n-1)),
$$

by Lemma $5 \mathrm{C}$ can be written as a product of commutators of the form

$$
\left[x_{i(1)}, y_{1}, \cdots, y_{t}, x_{i(2)}, \cdots, x_{i(3 n-3)}\right]
$$

where $t=2(m-n)+4 \geqq 8, x_{i(j)} \in\left\{x_{1}, \cdots, x_{m}\right\}$ and $|\{i(1), \cdots, i(3 n-3)\}| \leqq$ $n-1$. By Lemma 1 , each such commutator is trivial. This proves the lemma.

LEMMA 7 (GuPTA-GuPTA [3]). Let $G$ be a group of exponent 4 and let $n$ be a fixed positive integer such that for all $m \geqq 2$ every commutator of type $(m \rightarrow 2 m+n+1)$ is trivial. Then $G$ is solvable of bounded length. [3].)

(This lemma is an immediate corollary of the main theorem proved in

ProOf OF THE MAIN TheOREM. Let $\lambda(k) \rightarrow \infty$ as $k \rightarrow \infty$ and let $n \geqq 3$ be the least positive integer such that $\kappa(n) \leqq 3 n-3$. Let $F$ be the free group of exponent 4 of countably infinite rank. Since $\kappa(n) \leqq 3 n-3$, every commutator in $F$ of type $(n \rightarrow 3 n-2)$ is trivial. We shall prove that $F$ is solvable of bounded length which is contrary to the hypothesis.

Let $H$ be the group of exponent 4 generated by $a_{1}, a_{2}, \cdots$ and satisfying only the following relations and their consequences:

(I) $a_{i}^{2}=1, i=1,2, \cdots$.

(II) $\left[a_{i}, h, a_{i}\right]=1$ for all $a_{i}, i=1,2, \cdots$, and all $h$ in $H$.

In Gupta-Weston [2], it is shown that $F$ is solvable if and only if $H$ is solvable. Thus we may assume that in $H$ every commutator of type $(n \rightarrow 3 n-2)$ is trivial and conclude that $H$ is solvable. Let us assume further that $\gamma_{4}\left(\left\langle h^{H}\right\rangle\right)$ is trivial for all $h \in H$. By Lemma 6 , for $m \geqq 2$, every commutator of type $(m \rightarrow 2 m+n+1)$ is trivial and by Lemma $7, H$ is solvable of bounded length. Thus it remains for us to show that for all $h \in H, \gamma_{4}\left(\left\langle h^{H}\right\rangle\right)=\{1\}$. Let $K_{(i, n, h)}, n \geqq 1$, be the subgroup of $H$ generated by $a_{i(1)}, \cdots, a_{i(n)}$, and where $a_{i(1)}, \cdots, a_{i(n)} \in\left\{a_{1}, a_{2}, \cdots\right\}$. By Lemma 3 of [3], $\gamma_{n+4}\left(K_{(i, n, h)}\right)=\{1\}$. Since $\gamma_{4}\left(\left\langle h^{H}\right\rangle\right)$ is generated by all $\gamma_{n+4}\left(K_{(i, n, h)}\right)$ for $n=1,2, \cdots$, it follows that $\gamma_{4}\left(\left\langle h^{H}\right\rangle\right)=\{1\}$. This completes the proof of the main theorem. 


\section{BIBLIOGRAPHY}

1. N. D. Gupta and S. J. Tobin, On certain groups with exponent four, Math Z. 102 (1967), 216-226. MR 36 \#5220.

2. N. D. Gupta and K. W. Weston, On groups of exponent four, J. Algebra 17 (1971), 59-66.

3. C. K. Gupta and N. D. Gupta, On groups of exponent four. II, Proc. Amer. Math. Soc. 31 (1972), 360-362.

4. G. Higman, The orders of relatively free groups, Proc. Internat. Conf. Theory of Groups (Canberra, 1965), Gordon and Beach, New York, 1967, pp. 153-165. MR 36 \#2676.

5. A. L. Tritter, A module-theoretic computation related to the Burnside problem, Computational Problems in Abstract Algebra (Proc. Conf., Oxford, 1967), Pergamon, Oxford, 1970, pp. 189-198. MR 41 \#6981.

6. C. R. B. Wright, On the nilpotency class of a group of exponent four, Pacific J. Math. 11 (1961), 387-394. MR 23 \#A927.

Department of Mathematics, University of Manitoba, Winnipeg, Manitoba, CANADA

Department of Mathematics, University of Wisconsin-Parkside, Kenosha, WISCONSIN 53140. 